\title{
Positional uncertainty in long-term memory
}

\author{
JAMES S. NAIRNE \\ Purdue University, West Lafayette, Indiana
}

\begin{abstract}
Long-term memory for position was examined from the perspective of an immediate memory framework, the perturbation model of Estes (Estes, 1972; Lee \& Estes, 1977, 1981). First, a sim. ple version of the perturbation model is shown to provide a reasonable fit of previously reported long-term data (Nairne, 1990b), even though the perturbation idea was developed to explain the phenomena of immediate retention. Second, new results are reported that extend the application to multiple dimensions. Long-term memories for list and within-list position appear to mimic the classic patterns of immediate retention, in that both show bow-shaped serial position curves and error generalization gradients that are roughly symmetrical around the true serial position.
\end{abstract}

In a fundamental sense, the study of episodic memory is concerned with the encoding and retention of attributes along temporal and spatial dimensions. When items are forgotten from memory lists, it is not the items themselves that are forgotten, but rather their occurrences in prior spatiotemporal windows. Over the past two decades, studies of immediate retention have revealed that memory for temporal and spatial position information changes in a systematic fashion over time. In particular, a subject's knowledge about position becomes less precise, leading to inversion errors in ordered recall and recognition, in a manner described by regular position-based generalization gradients (see, e.g., Bjork \& Healy, 1974; Estes, 1972; Jahnke, Davis, \& Bower, 1989; Lee \& Estes, 1977, 1981). The empirical analysis of these gradients, termed positional uncertainty distributions, led to the development of perturbation theory (Estes, 1972; Lee \& Estes, 1977,1981 ), which has proven to be a highly successful descriptor of immediate memory phenomena (see Estes, 1987, for a review).

In the present article, the logic of perturbation theory is extended into a long-term memory setting. The impetus for the work was a recent finding that serial position errors after $10 \mathrm{~min}$ of distraction appear to mimic, in most respects, the generalization gradients typically obtained in immediate retention (Nairne, 1990b). In both the immediate and the delayed case, if subjects place an item in the wrong serial position at test, error position tends to cluster around the true serial position and to decline gradually as distance from the true position increases. First, a simple version of the perturbation model will be shown to provide a reasonable fit of the long-term data, even though the perturbation idea was developed initially as a model of immediate retention. Second, new data are

Thanks are due to Addie Dutta for helpful comments throughout the research, and to Professors Estes, Healy, and Intons-Peterson for valuable comments on an earlier version of the manuscript. Correspondence concerning this research should be addressed to James S. Naime, Department of Psychology, Purdue University, 1364 Psychology Building, Room 3152, West Lafayette, IN 47907. reported from an investigation of long-term memory for position along multiple dimensions: After several minutes of distraction, subjects were required to remember not only an item's position within a list, but also the particular list in which the item had occurred. For both the list and the position dimensions, performance will be shown to follow the patterns anticipated by the perturbation model.

\section{The Theoretical Perspective}

The manner in which serial position information is lost over time, as indexed by the error position gradients, is the important ingredient of the perturbation model. Following Lee and Estes (1981), the presentation of an event (e.g., a list item) can be assumed to lead to an initial representation of values at a number of different levels, or occurrence dimensions (also see Huttenlocher, Hedges, \& Bradburn, 1990). For example, a typical memory experiment might be represented in three dimensions, Exp $[i][j][k]$, where variable $[i]$ maps the order of lists as presented, variable $[j]$ the order of subjective groupings within a list, and variable $[k]$ the position of the item within a group. Thus, a particular entry in the array, Exp[3][2][1], stands for the first item in the second group of the third list in the experiment. Conceptually, the encoded event can then be viewed as occupying a position in a multidimensional space, where each dimension stands for an ordered variable defined by the experimental context (i.e., list, group, position).

Of primary interest is the question of how the encoded item's position in this multidimensional space changes as a function of time. During forgetting, each of the originally encoded values loses precision through a perturbation process, in which encoded values swap positions with neighboring values along their respective dimensions. Forgetting is thereby characterized not in terms of an absolute loss of information, as in a decay model, but rather as movement in the multidimensional space. As a result, there is a probability that an item will lose its originally encoded value, and another probability that it will recover it at some later time. The amount of movement expected is stochastic, and it is constrained by several factors. First, 
each dimension is assumed to have its own perturbation rate, which establishes the likelihood that any swapping of position values will occur. Second, the experimental task demands will establish the range of values that the dimension can adopt, and consequently the amount of possible movement. For instance, if there are only three lists in the experiment, then items can possess only three values along the list dimension. Or, if the subject is aware that the second list contains only birds, then little movement would be expected along the list dimension for items that are birds. Third, when movement is equally likely in either direction-a common but not essential assumption of the model-then items that occupy the endpoints of their respective dimensions will show some initial inertia. With each tick of the clock, these values will be less likely to drift because they can leave their position only in one direction, inward toward intermediate values; this limiting factor has been proposed to underlie primacy and recency effects in immediate serial recall (Estes, 1972).

By way of illustration, assume that we are dealing with only a single dimension, the position of an item within a single, ungrouped list. The probability that an item will occupy a particular interior position $i$ at time $n+1$ can be expressed as

$$
X_{i, n+1}=(1-\theta) X_{i, n}+(\theta / 2) X_{i-1, n}+(\theta / 2) X_{i+1, n},
$$

where $\theta$ is the probability that the item will suffer a perturbation, causing it to adopt the position of a neighboring item. In other words, the likelihood that an item will occupy, say, the third position in the list at time $n+1$ is equal to the probability that it was in Position 3 at time $n$, and no perturbation occurred $(1-\theta)$, plus the probabilities that it was in either Position 2 or Position 4 at time $n$ and perturbations did, in fact, occur $(\theta / 2$ assumes movement is equally likely in either direction). The equations for items occupying endpoints of the dimension (assuming endpoints are possible) are slightly different. Here is an example:

$$
X_{1, n+1}=[1-(\theta / 2)] X_{1, n}+(\theta / 2) X_{2, n} .
$$

In this case, the probability that an item will leave an endpoint position is $\theta / 2$ because it is only possible to leave in one direction. These simple functions allow one to specify, as a function of time, the likelihood that an encoded event will occupy any of the possible dimensional values; as noted previously, the resulting theoretical gradients have been shown to mimic the empirical uncertainty gradients quite well (see, e.g., Lee \& Estes, 1977).

\section{Fits to the Long-Term Case}

The perturbation model was developed to account for the data of immediate retention, but recent data from my laboratory (Nairne, 1990b) indicated that it might be applied successfully to a long-term memory setting. In each of a series of experiments, subjects were asked to give pleasantness ratings about words in short lists, under the instructional guise of making "rapid judgments about words." The items were high-frequency nouns, presented aloud on a tape recorder, with $2.5 \mathrm{sec}$ separating the onset of each word from the onset of the next. Typically, five lists of five words were presented, with a 5-sec blank interval separating the lists. The subjects were told that they would be rating five lists of five words, but they were given no information about why the words were grouped in this way or about the final memory test. Following a distractor interval of approximately 10 min (finding car names hidden in a matrix of letters), a sheet of paper containing the rated lists was handed out, but with the words for each list reordered randomly. The subject's task was to reconstruct the original order of presentation for each list. Reconstruction tasks of this type can be seen as pure tests of position memory, or possibly of order memory, because the critical items are made available at the time of test (see Healy, 1982).

Data from Nairne (1990b) are displayed in Figure 1 (the filled squares), plotted as a function of the serial position of the item in a list. ${ }^{1}$ The results have been collapsed across the five lists, and each data point represents the mean correct positioning of an item in its proper serial position for the reconstruction task. Performance was quite good after $10 \mathrm{~min}$, and the bow-shaped character of the serial position curve mimicked the usual finding in immediate retention (see Bjork \& Healy, 1974; Healy, 1974). The open squares in the same figure are the predictions from the perturbation model, as provided by recursive applications of Equations 1 and 2. The perturbation rate, $\theta$, was set at 0.15 -the same value that was used

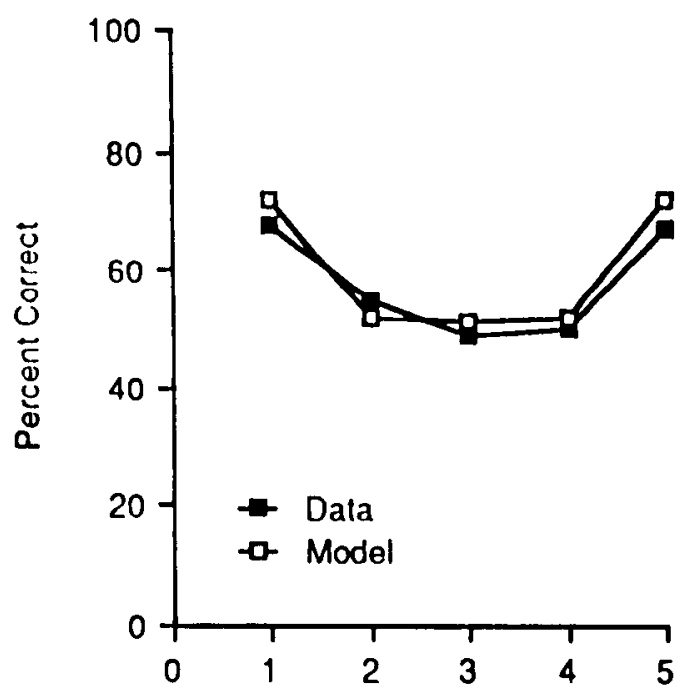

Serial Position

Figure 1. Serial position curves for the data from Nairne (19\%0b; filled squares) and predicted values from applications of the perturbation equations $(\theta=0.15$; open squares). [The data are from the dissimilar group in Experiment 2 of "Similarity and long-term memory for order," by J. S. Nairne, 1990, Journal of Memory \& Language, 29, 733-746. Copyright 1990 by Academic Press. Adapted by permission]. 
by Lee and Estes (1977)-and the number of perturbation opportunities was set equal to the number of items in the list. Consequently, the predicted points portray the probabilities that each item will be in its proper serial position after five recursive passes through the equations. Although the fit is not perfect, it captures the essential empirical trends quite well.

Even more diagnostic are the positional uncertainty curves, which are displayed in Figure 2. The curves in the bottom half of the figure are from the actual data, and the upper half of the figure shows the predicted uncertainty curves from the perturbation model. For each serial position, the data display the classic generalization gradients that are the hallmarks of immediate memory for order (see, e.g., Bjork \& Healy, 1974; Estes, 1972; Jahnke et al., 1989; Lee \& Estes, 1977). When an error was made, subjects were more likely to place the presented item in a location adjacent to its correct one, and error position declined gradually with increasing distance from the presented position. The simulated position functions, which were based on the same parameter values as were the data of Figure 1, correspond closely in general form to the actual data, even though the data were collected after $10 \mathrm{~min}$ rather than in immediate retention.

Moreover, as in immediate memory, these position functions can be used to predict the likelihood that subjects will commit inversion errors in reconstruction. Spe- cifically, if reconstruction performance is governed solely by memory for position, then subjects should be likely to place two items in the wrong relative order to the extent that the positional uncertainty curves for those two items overlap (cf. Lee \& Estes, 1977; Jahnke et al., 1989). These predictions are presented in Table 1 , along with the actual inversion percentages (i.e., where a given pair of serial positions was placed in the wrong relative order by the subjects). The theoretical values are based on the empirical position gradients, assuming independence, and were calculated by summing the probabilities that, say, Position 2 would fall to the left of Position 1 for each of the possible serial positions. Although in each case the model predicts that fewer inversion errors should have occurred, thereby leading one to reject the idea that test performance is governed completely by independent memories for position, the rank orderings of predicted and observed values are strikingly close (e.g., a Pearson $r$ between predicted and observed yields a correlation coefficient of .95).

\section{UNCERTAINTY ALONG MULTIPLE DIMENSIONS}

In earlier work on this topic (Nairne, 1990b), subjects received the items at test, reordered as lists. It has not been necessary, therefore, to remember the particular
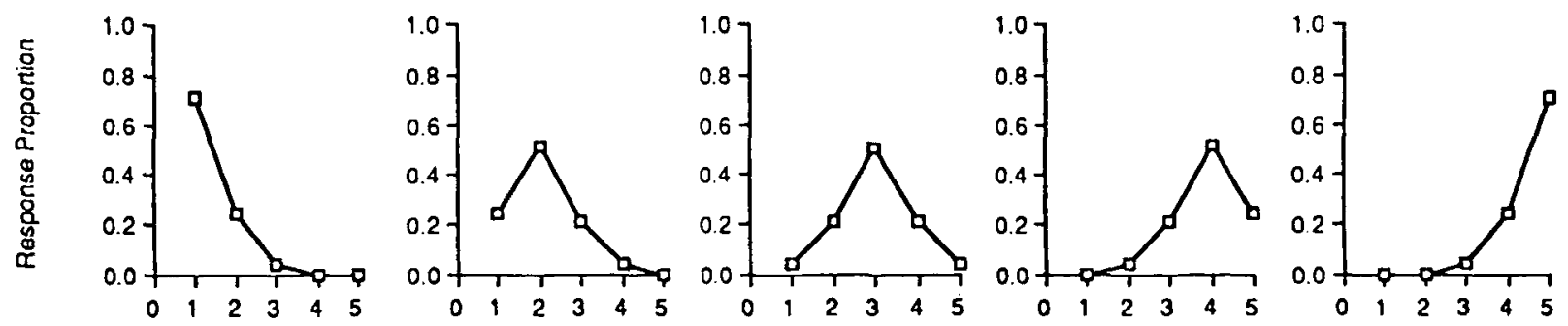

\section{Serial Position}

\section{Data}
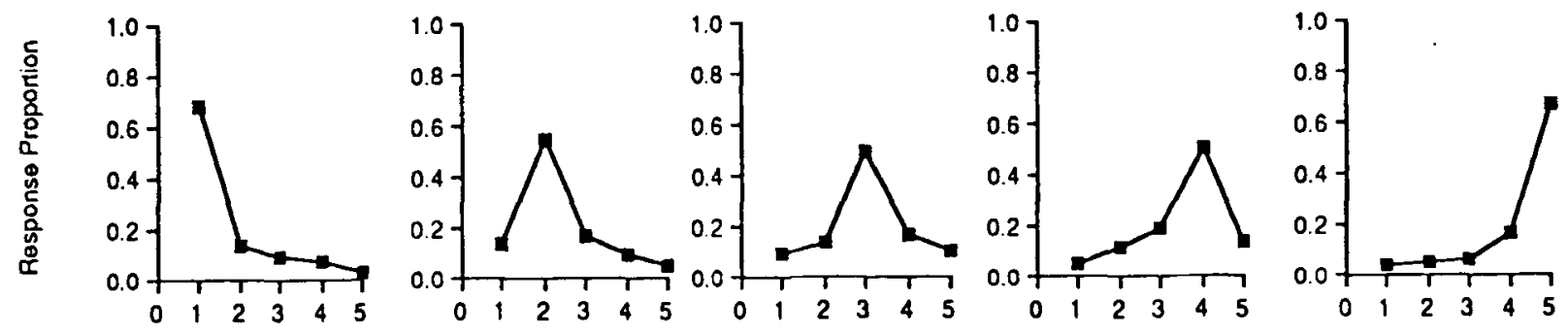

Serial Position

Figure 2. The positional uncertainty curves for the data (bottom half) and those predicted from the perturbation model (upper half). The panels from left to right represent the presented Serial Positions 1-5. 
Table 1

Inversion Percentages for Pairs of Ordingl Pocitions in Nairne (1990) Compared with Predictions from the Position Functions

\begin{tabular}{ccc}
\hline $\begin{array}{c}\text { Ordinal } \\
\text { Position }\end{array}$ & Observed & Predicted \\
\hline 1,2 & .21 & .18 \\
2,3 & .26 & .20 \\
3,4 & .38 & .25 \\
4,5 & .24 & .14 \\
1,3 & .19 & .12 \\
1,4 & .14 & .07 \\
1,5 & .10 & .03 \\
2,4 & .19 & .13 \\
2,5 & .11 & .07 \\
3,5 & .17 & .11 \\
\hline
\end{tabular}

items that may have occurred, or how the items were grouped together as lists. In the present experiment the latter constraint was relaxed by requiring that items be placed at test in their proper lists as well as by position within a list. The subjects were asked to produce pleasantness ratings about five words in each of five lists. Following 2 min of distraction, each subject was handed a test sheet containing five rows of five blanks with the 25 rated words reordered randomly at the bottom of the page. The subjects were required to place each item first in its proper list (i.e., row of blanks) and then in its proper position within the chosen list.

Two kinds of uncertainty distributions can be generated from these data, one at the level of lists and one at the level of position within a list; in each case, the shapes of the position generalization gradients should look similar. For example, when a subject places an item in an incorrect list, the chosen list should be near the correct one, and errors should fall off with increasing distance from the true list (as with position within a list). Moreover, if forgetting along the list and within-list position dimensions proceeds independently, then when subjects place an item in the wrong list, one might expect them to place that item in its proper within-list serial position. Similar findings have been obtained in list recognition experiments by Hintzman, Block, and Summers (1973), and in immediate memory experiments, through an analysis of intrusion errors, by Wickelgren (1967).

\section{Method}

\section{Subjects}

The subjects were 33 undergraduates who participated for course credit. The experimental sessions were conducted in groups of 5-15 subjects and lasted approximately $20 \mathrm{~min}$.

\section{Design and Materials}

To enhance generality, three different versions of the experiment were conducted, with completely different word lists used in each case. Each replication comprised 25 words, arranged in five lists of 5 words. The stimulus words were medium- and high-frequency nouns, four to six letters in length, drawn from the Paivio, Yuille, and Madigan (1968) norms. The mean imagery value for the words was 5.79 (the range was 2.20 to 6.90) and the average meaningfulness rating was 6.53 (the range was 5.04 to 8.32 ). Assignment of the words to lists and positions within lists was determined randomly by sampling without replacement from the overall pool of 75 words. The subjects were placed in a particular version of the experiment, depending on their respective sign-up times. Within each group, all subjects received the same lists in the same presentation order.

\section{Procedure}

The stimulus items were presented aloud on a tape recorder with 2.5 sec separating the onset of each word from the onset of the next word. Each of the five lists began with the word "ready" and ended with a 5-sec blank interval. The subjects were told to make a pleasantness rating for each word as it was presented, on a scale ranging from 1 (unpleasant) to 3 (pleasant). Everyone was told to respond quickly because the experiment was concerned with first impressions about the pleasantness of words. No information was given about the later memory test, or about why the words were grouped in this fashion. The subjects were led to believe that they were participating in a very simple rating task. The rating responses were written on a response sheet that contained five blanks listed next to each of the numbers $1-5$.

After all 25 words had been rated by the subjects, they were handed a distractor task that required them to solve math problems. After approximately $2 \mathrm{~min}$, these sheets were collected and the reconstruction test was administered. Each of the test sheets contained five rows of five blanks listed next to the numbers 1-5. At the bottom of the page, the 25 rated words were listed in three rows of 8,8 , and 9 items, respectively. Assignment of the words to these three lists was determined randomly. The subjects were told to place each of the words listed at the bottom of the page first in its proper list (as determined by the particular row of response blanks), and then in its proper position within the list. Everyone was told to be careful about using the same word twice, and was advised to cross out a word at the bottom of the page after it had been used. The subjects were given as much time as they needed to complete the reconstruction test.

\section{RESULTS AND DISCUSSION}

There are a number of ways to present the results of the reconstruction test, but it will be instructive initially to examine how weil subjects were able to reconstruct values of one dimension without regard to performance on the other. The left side of Figure 3 displays percent correct reconstruction performance separately for the list and position dimensions. The data have been collapsed across replications, because each version of the experiment resulted in the same empirical trends.

The list curve shows how well subjects were able to place items in their appropriate lists, irrespective of where in the list the item happened to be placed, as a function of list serial position. For the position curve, items were scored as correct if they were placed in the appropriate within-list serial position, regardless of list placement.

In both cases, one finds bow-shaped serial position curves of the type usually obtained in the immediate retention of temporal order (see, e.g., Healy, 1974). The subjects were more likely to place an item in its appropriate list if the items occurred initially in either the first or the last list, and performance levels were lower, and roughly comparable, for the interior lists. Similar findings have been obtained in long-term memory studies of list membership (e.g., Hintzman et al., 1973; Underwood, 


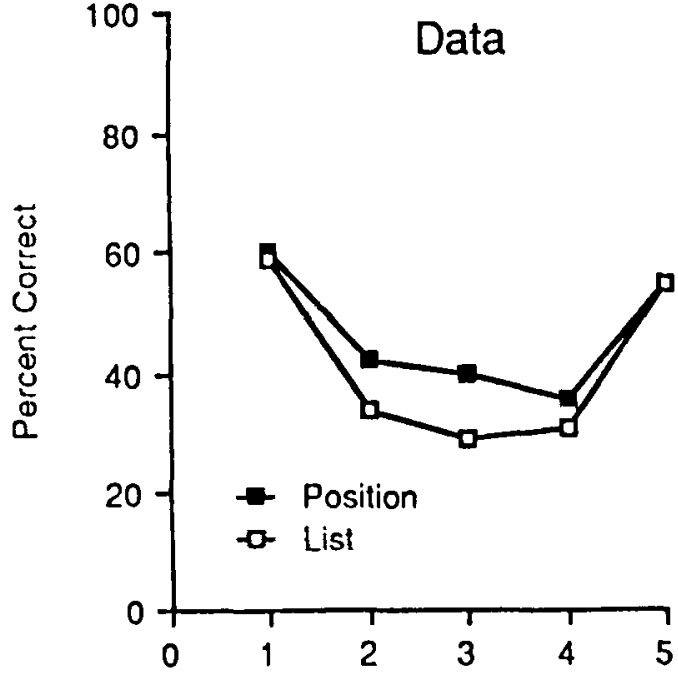

Serial Position

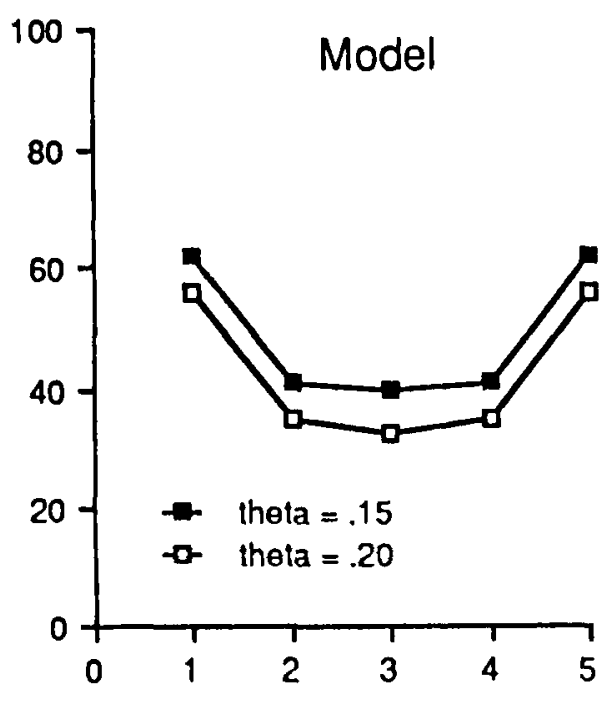

Serial Position

Figure 3. Serial position curves for the list and within-list position dimensions. The right side of the figure shows simulated curves based on perturbation rates of 0.20 and 0.15 .

1977). Essentially the same pattern was found for the position curve: Subjects were better able to place items from either the first or the last positions correctly within a list, and performance was lower for the middle serial positions. The latter result replicates earlier work in this laboratory (Nairne, 1990b), although in the present case the subjects were given considerably less information at test. There is also some indication in the data that the subjects possessed better memory for within-list position values, in comparison with list membership values, but the main effect of dimension (list vs. within-list position) was not significant in an analysis of variance $[F(1,64)=$ $2.42, M S_{c}=2.32$ ]. In contrast, as expected, there was a highly reliable main effect of serial position $[F(4,256)$ $=32.86, M S_{\mathrm{e}}=.740 \mathrm{]}$, and there was no reliable interaction of dimension $\times$ serial position $[F(4,256)=1.07$, $\left.M S_{\mathrm{e}}=.740\right]$.

The finding of bow-shaped serial position curves for both the list and the position dimensions follows naturally from the theoretical analysis proposed earlier. If one assumes that represented dimensional values, regardless of the dimension in question, lose precision in accordance with a perturbation-like process, then values at the endpoints should be retained better. Items that occupy the endpoint positions can leave their positions in only one direction, so they should be more resistant to drifting over time. To make this point more concretely, some further simulations of the perturbation model are shown on the right side of Figure 3. These curves, as earlier, were obtained through recursive applications of Equations 1 and 2 and are meant to stand, roughly, for the list and position curves obtained from the actual data. The filled squares were obtained with $\theta$ set at 0.15 , and with eight recursive applications of the equations; the open squares were obtained with the same number of applications, but with a higher perturbation rate $(\theta=0.20) .{ }^{2}$ There are differences between the data and the predictions of the model, but the simulated curves show once again the marked primacy and recency effects that are characteristic of the immediate and long-term data.

\section{Overall Performance}

At this point, we can examine subject performance overall and assess how well it can be predicted from considering performance on each dimension alone. It seems possible that subjects decide on their responses by separately accessing the encoded values along each dimension. For example, a subject might first decide the appropriate list, and then, on the basis of retention of the within-list dimensional value, decide where in the chosen list to place the response. If these two values are retained independently, the probability of being correct overall, in the simplest case, should be equal to the product of the probabilities that the list and within-list position values are retained correctly. Percent correct retention performance is shown in Table 2, as a function of within-list serial position. In this case, it was necessary for the subject to place an item in its proper list and within-list position in order for a response to be counted as correct. The same table also shows the predicted values formed from computing the product of the average values for the list and within-list position dimensions for either the actual data shown on the left side of Figure 3 (Predicted ${ }_{D}$ ) or the simulated data shown on the right side of the same figure $\left(\right.$ Predicted $\left._{M}\right)$. The predicted values correspond quite closely to the actual data for the first and last serial positions, but subjects performed better than predicted for the interior positions. Further evidence relevant to the issue of independence 
Table 2

Overall Percent Correct Performance and Predicted Values from Simulated and Actual List and Position Performance

\begin{tabular}{llllll}
\hline & \multicolumn{5}{c}{ Serial Position } \\
\cline { 2 - 5 } & 1 & 2 & 3 & 4 & 5 \\
\hline Data $_{\text {Predicted }}$ & .35 & .25 & .15 & .20 & .33 \\
Predicted $_{M}$ & .35 & .14 & .12 & .11 & .30 \\
\hline
\end{tabular}

can be gathered from examination of the uncertainty gradients for each dimension, which are described in the next section.

\section{The Uncertainty Gradients}

Of most interest are the generalization gradients, representing how responses were distributed across the various positions at test. These results are shown in Figures 4 and 5, separately for the list and position dimensions. Each panel shows response proportions for a given list, or position within a list, across the possible position placements. In each case, the gradients have been further conditionalized in terms of whether or not the subject retained the correct value of the corresponding dimension. For example, in the upper half of Figure 4, the within-list generalization gradients are shown for items that subjects placed correctly in the appropriate list; in the bottom half of the figure, the gradients are shown for conditions in which subjects placed the item in the wrong list. Figure 5 shows how the list membership responses were distributed, conditionalized in terms of whether the item was placed in the correct or incorrect within-list serial position.

As noted earlier, when errors are made in immediate retention they tend to cluster around the true position and decline gradually with increasing distance away from the correct position. For both the list and the position dimensions, the data resemble the pattern found in immediate memory studies. The position gradients replicate the results of Nairne (1990b), and the list gradients provide important support for the generality of the forgetting process. When subjects placed an item in an incorrect position, the chosen position tended to be near the correct one, and fewer incorrect placements were made in temporally distant positions; this characterization of the results applies equally well for the list and within-list position dimensions and, in virtually every case, it applies regardless of whether or not the subject was correct on the other dimension. As argued by Lee and Estes (1977), forgetting does not seem to be an all-or-none process, but rather represents a loss in certainty about the originally encoded value.

Although position-based generalization gradients are apparent in each of the panels in Figures 4 and 5, there are

Correct List
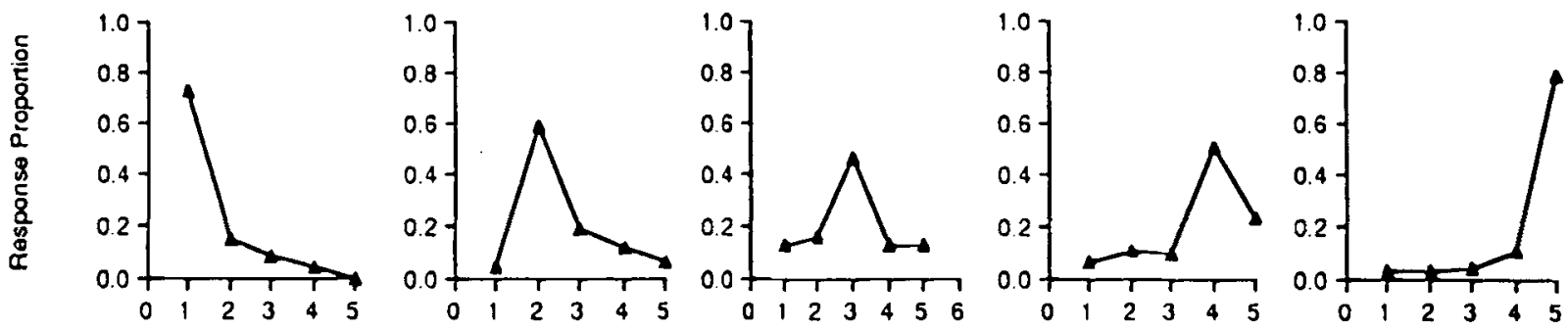

Within List Position

Incorrect List
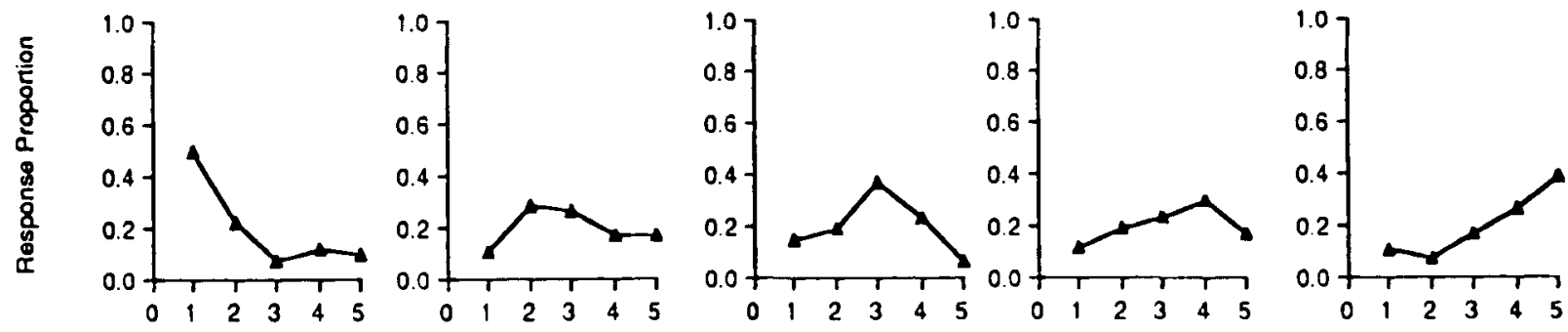

Within List Position

Figure 4. Within-list positional uncertainty curves for items that were placed in the correct (upper haln) and incorrect (lower haln) lists at test. The panels from left to right represent the presented within-list Serial Positions $1-5$. 
Correct Position
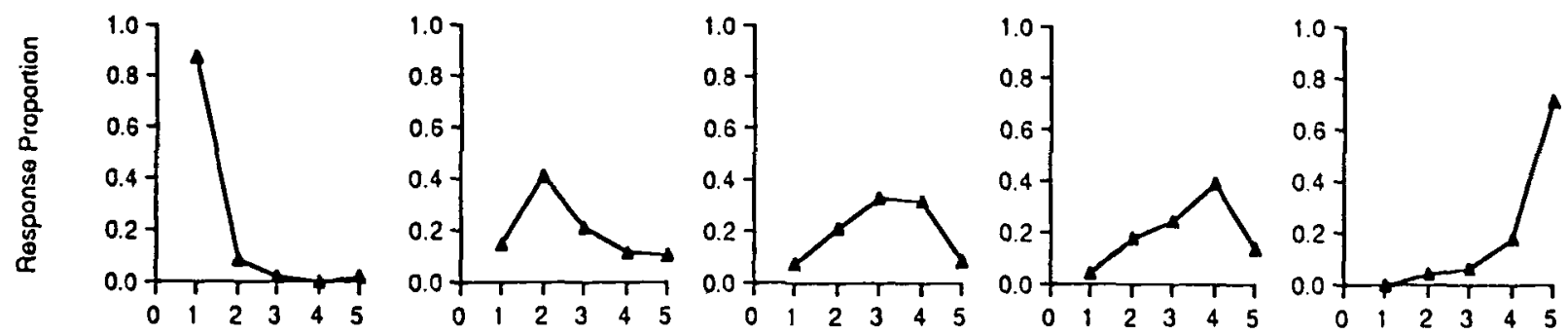

List Position

Incorrect Position
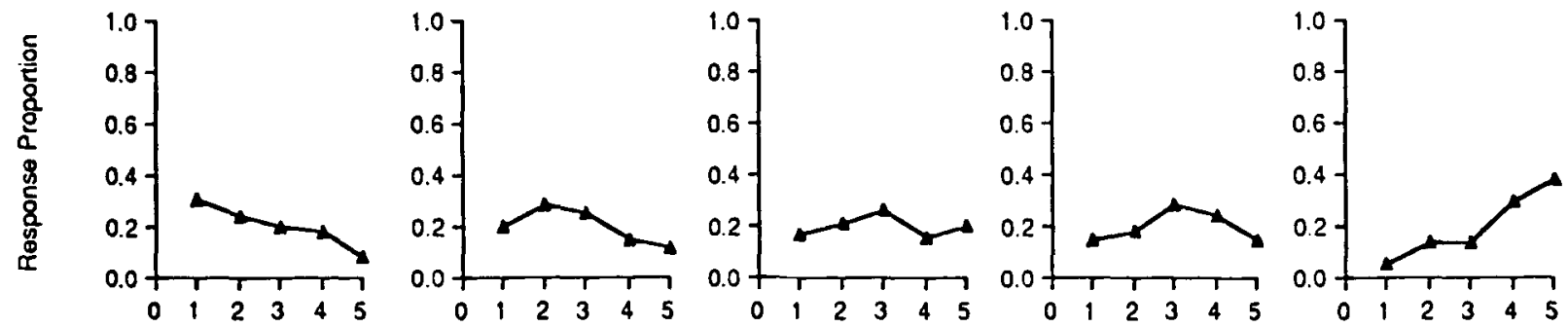

List Position

Figure 5. List positional uncertainty curves for items placed in the correct (upper half) and incorrect (lower half) within-list positions at test. The panels from left to right represent the presented List Positions 1-5.

clear differences between the upper and lower halves of each figure. If knowledge about the list and within-list position dimensions was retained independently, then one would have expected little, if any, absolute differences from conditionalizing performance of one dimension on the basis of the other. For true independence, the probability of placing an item in, say, the correct within-list position ought to be the same regardless of list placement. This was not the case. For both the list and within-list position dimensions, subjects were more likely to make the correct placement if they also retained the correct value on the other dimension. To confirm these trends statistically, overall analyses of variance were performed on the proportion of responses that fell in the correct position as a function of serial position and correct or incorrect retention of the other dimension. For the list dimension, subjects showed reliably better list judgments when they were correct on the within-list dimension $[F(1,32)=$ 39.95, $M S_{\mathrm{e}}=.113$ ], a main effect of list serial position $\left[F(4,128)=14.12, M S_{\mathrm{e}}=.076\right]$, and a significant interaction of serial position $\times$ performance on the withinlist dimension $\left[F(4,128)=6.19, M S_{\mathrm{e}}=.132\right]$. The analyses on the within-list dimension showed a similar pattern: Reliable effects were found for correctness on the list dimension $\left[F(1,32)=36.48, M S_{e}=.105\right]$, for within- list serial position $\left[F(4,128)=11.85, M S_{\mathrm{e}}=.081\right]$, and for the interaction $\left[F(4,128)=2.98, M S_{\mathrm{e}}=.125\right]$.

Although these data, coupled with the analyses presented in Tables 1 and 2, lead one to reject true independence, the fact that position-based generalization gradients are obtained regardless of performance on the other dimension has important implications for theory. ${ }^{3}$ Even if subjects incorrectly retained the value of one dimension, they were still most likely to respond with the correct value on the other dimension. This finding provides strong support for the assumption that subjects possess separate indices for each dimension, and are probably not performing on the basis of a single index, such as a time tag. For example, if subjects placed the first item of the third list incorrectly in the second list, then a unidimensional time tag analysis would predict that the item should be placed near the end of the second list; that is, the last item in the second list is closest in time to the original position. Instead, subjects were most likely to place the item in the first serial position of the second list, counter to the prediction of the time tag analysis (see Hintzman et al., 1973, for similar reasoning). It is more parsimonious to assume that subjects encoded and retained separate information about list and position, which they combined, in some fashion, at the point of test. 


\section{CONCLUSIONS}

These results, combined with the examination of the long-term memory data of Nairne (1990b), provide support for the application of an immediate memory perspective to the analysis of long-term memory for order and position. Performance in the long-term case clearly mimics, in many respects, performance in immediate memory, although this fact is not often recognized among memory researchers. In both cases, the retention of temporal order produces bow-shaped serial position curves and error gradients that are approximately symmetrical around the true position. In the present experiment, these empirical principles were extended to multiple dimensions; it was shown that bow-shaped serial position curves and symmetrical error gradients apply to list as well as to within-list position judgments. As I have argued elsewhere (Nairne, 1990a, 1990b), explication of these empirical trends will certainly be an important ingredient of any successful general model of order retention.

With regard to perturbation theory, in the study of immediate retention researchers have typically assumed that perturbations arise as a consequence of rehearsal-based recodings that are needed to keep information active over the short term (see Cunningham, Healy, \& Williams, 1984; Healy, Fendrich, Cunningham, \& Till, 1987; Lee \& Estes, 1981). Long-term retention of order and position information, in contrast, was presumed to be mediated by accessing a frozen or crystallized structure in which perturbation rates had fallen to zero (perhaps due to the development of inhibitory associations; see Estes, 1972). It is possible that the data of the present experiment are due, in part, to the accessing of such a crystallized structure. However, there is no clear reason why the logic of perturbation theory could not be applied to any time frame, including the study of how position information might be lost over minutes, hours, or days. It simply becomes a matter of specifying the perturbation rates, and of dealing with the question of whether the perturbation process operates in relative or absolute time.

There have been few systematic attempts in the laboratory to document how uncertainty about temporal position changes over retention intervals longer than a minute or so, but relevant data have been collected in more naturalistic settings. Baddeley, Lewis, and Nimmo-Smith (1978), who had subjects report the date of their last participation in a laboratory experiment, found that uncertainty about the date, as measured by the absolute error, increased regularly with elapsed time (see also Rubin \& Baddeley, 1989; Thompson, 1982). Such a finding is consistent with the perturbation idea, which also predicts that average error distance should increase systematically as more opportunities for perturbations arise. The recent models of Rubin and Baddeley (1989) and Huttenlocher et al. (1990) on the naturalistic retention of occurrence information (e.g., "What was the exact date that you last ate fish?") also incorporate the important assumption that temporal uncertainty grows regularly with time. Like Lee and Estes (1981), Huttenlocher et al. (1990) assume that correct recall of temporal position information is mediated by accessing values along occurrence dimensions that reside at multiple levels (e.g., calendar time: days, months, years, etc.). In the naturalistic case, however, as the encoded values become more inexact, subjects introduce systematic biases into their estimates. For instance, knowledge about boundaries ("I know it occurred during the academic year') can lead to memory telescoping in which events are reported as having occurred more recently than they actually did (Loftus \& Marburger, 1983; Rubin \& Baddeley, 1989). In addition, subjects tend to report prototypical values for their estimates (e.g., dates differing by weeks or months), perhaps as a way of compensating for the inexactness of the recovered value.

There is clearly an important need to pursue integration of the theoretical frameworks that have been designed to handle data in the short- and long-term memory literatures. If immediate retention and delayed retention really reflect the operation of a single, but perhaps complex, set of memory laws, then it is natural to expect theories that work well in one context to work reasonably well in the other. For a number of reasons, perhaps the most significant of which is the belief in separate short- and long-term memory stores, these extensions have rarely been tried. In the current instance, a variant of an existing short-term memory perspective, perturbation theory (Estes, 1972; Lee \& Estes, 1977, 1981), has been used to generate empirically testable predictions about longterm retention, and to provide a framework for the subsequent interpretation of the findings.

\section{REFERENCES}

Baddeley, A. D., Lewis, V., \& Nimmo-Smith, I. (1978). When did you last ...? In M. M. Gruneberg, P. E. Morris, \& R. N. Sykes (Eds.), Practical aspects of memory (pp. 73-83). London: Academic Press.

Buork, E. L., Healy, A. F. (1974). Short-term order and item retention. Journal of Verbal Learning \& Verbal Behavior, 13, 80-97.

Cunningham, T. F., Healy, A. F., Williams, D. M. (1984). Effects of repetition on short-term retention of onder information. Journal of Experimental Psychology: Learning, Memory, \& Cognition, 10. 575-597.

EsTES, W. K. (1972). An associative basis for coding and organization in memory. In A. W. Melton \& E. Martin (Eds.), Coding processes in human memory (pp. 161-190). Washington, DC: Winston.

EsTEs, W. K. (1987). One hundred years of memory theory. In D. S. Gorfein \& R. R. Hoffman (Eds.), Memory and leaming: The Ebbinghaus Centennial Conference (pp. 11-33). Hillsdale, NJ: Erlbaum.

HealY, A. F. (1974). Separating item from order information in shortterm memory. Journal of Verbal Learning \& Verbal Behavior, 13, 644-655.

HeALY, A. F. (1982). Short-term memory for order information. In G. H. Bower (Ed.), The psychology of learning and motivation (Vol. 16, pp. 191-238). New York: Academic Press.

Healy, A. F., Fendrich, D. W., Cunningham, T. F., \& Till, R. E. (1987). Effects of cuing on short-term retention of order information. Journal of Experimental Psychology: Learning, Memory, \& Cognition, 13, 413-425.

Hintzman, D. L., Block, R. A., \& Summers, J. J. (1973). Contextual associations and memory for serial position. Joumal of Experimental Psychology, 97, 220-229. 
Huttenlocher, J., Hedges, L., \& Bradburn, N. (1990). Reports of elapsed time: Bounding and rounding processes in estimation. Journal of Experimental Psychology: Learning, Memory, \& Cognition, 16, 196-213.

Jahnke, J. C., Davis, S. T., Bower, R. E. (1989). Position and order information in recognition memory. Joumal of Experimental Psychology: Leaming, Memory, \& Cognition, 15, 859-867.

LeE, C. L., Estes, W. K. (1977). Order and position in primary memory for letter strings. Journal of Verbal Learning \& Verbal Behavior, 16, 395-418.

LEE, C. L., EsTEs, W. K. (1981). Item and order information in shortterm memory: Evidence for multilevel perturbation processes. Journal of Experimental Psychology: Human Leaming \& Memory, 7, 149-169.

LofTus, E. F., MARBURGer, W. (1983). Since the eruption of Mt. St. Helens, has anyone beaten you up? Improving the accuracy of retrospective reports with landmark events. Memory \& Cognition, 11, 114-120.

NAIRNE, J. S. (1990a). A feature model of immediate memory. Memory \& Cognition, 18, 251-269.

NAIRNE, J. S. (1990b). Similarity and long-term memory for order. Journal of Memory \& Language, 29, 733-746.

Paivio, A., Yuille, J. C., \& Madigan, S. A. (1968). Concreteness, imagery, and meaningfulness values for 925 nouns. Journal of Experimental Psychology Monographs, 76(1, Pt. 2).

Rubin, D. C., \& BAdDeley, A. D. (1989). Telescoping is not time compression: A model of the dating of autobiographical events. Memory \& Cognition, 17, 653-661.

Thompson, C. P. (1982). Memory for unique personal events: The roommate study. Memory \& Cognition, 10, 324-332.

UNDERWOOD, B. J. (1977). Temporal codes for memories: Issues and problems. Hillsdale, NJ: Erlbaum.
WickELGREN, W. A. (1967). Rehearsal grouping and hierarchical organization of serial position cues in short-term memory. Quarterly Journal of Experimental Psychology, 19, 97-102.

\section{NOTES}

These data are from the dissimilar condition in Experiment 2 of the Nairne (1990b) study. They were chosen, in part, because subjects were tested on five five-word lists, as in the current study. Overall, however, these data are quite representative of reconstruction performance in this paradigm (e.g., see the serial position curves in Figure 3 of the present study).

2. I can offer no good rationale, beyond obtaining a better fit, for changing the number of recursive applications of the perturbation equations from five (Figure 1) to eight (Figure 3). Within-list position performance is not as accurate in the present study, but less information was given to the subjects at test. At some point, a consistent application rule will need to be worked out for the long-term case.

3. Independence analyses are further complicated by the fact that there might be third factors responsible for the detected dependencies. For example, if the processing records for some lists are simply accessed better at test, one would expect better list and within-list position performance for the items in those lists. In this sense, the curves in the bottom rows of Figures 4 and 5 might simply reflect processing records that have undergone more perturbations.

(Manuscript received October 26, 1990; revision accepted for publication January 2, 1991.)

\title{
Notices and Announcements
}

\author{
Members of Underrepresented Groups: \\ Reviewers for Journal Manuscripts Wanted
}

On behalf of Memory \& Cognition and Psychonomic Society Publications, I invite you to contact me if you are interested in reviewing manuscripts for Memory \& Cognition. Please send a letter and a copy of your curriculum vita to me at the following address: Memory \& Cognition, Department of Psychology, Indiana University, Bloomington, Indiana 47405. The letter or the vita should contain your complete address (including an electronic mail address if one is available), telephone number, and area(s) of expertise. Our reviewers have published articles in peer-reviewed journals, a standard prerequisite for being selected as a reviewer.

Please note that reviewing manuscripts takes time and must be completed quickly. If you are asked to review a manuscript, you will be expected to provide a thorough and prompt review. 